\title{
The performance of Gauss Markov's mobility model in emulated software defined wireless mesh network
}

\author{
Tsehay Admassu Assegie, Pramod Sekharan Nair \\ Department of Computing Technology, College of Engineering and Technology, Aksum University, Ethiopia
}

\begin{tabular}{l}
\hline \hline Article Info \\
\hline Article history: \\
Received Aug 29, 2019 \\
Revised Nov 1, 2019 \\
Accepted Nov 11, 2019 \\
\hline Keywords: \\
Gauss markov mobility model \\
Network performance \\
Software defined wireless \\
network \\
Wireless network emulation \\
Wireless node mobility \\
\hline
\end{tabular}

\begin{abstract}
A software defined wireless mesh network is an emerging trend in wireless communication promising better flexibility, reliability, and performance over traditional wireless local area network. And node mobility is the prominent feature of the next generation wireless mesh network. Mininet-wifi is a test bed investigation and emulation tool that plays an important role in the evaluation of wireless network's node mobility models such as, Gauss Markov and RandomWayPoint. This paper is focused on the Gauss Markov's wireless node mobility model and simulation of the model's performance within an emulated software defined wireless mesh network. Finally, the paper presents the performance of the model using the mininetWi-Fi emulator.
\end{abstract}

Copyright $\odot 2020$ Institute of Advanced Engineering and Science. All rights reserved.

Corresponding Author:

Tsehay Admassu Assegie,

Department of Computing Technology,

Aksum University, Ethiopia.

Email: tsehayadmassu2006@gmail.com

\section{INTRODUCTION}

Mobile communication permits a device to communicate with other devices in a wireless mesh network regardless of their movement form one coverage area of network access point to the other [1]. In order to maintain the connectivity between the moving nodes in a wireless mesh network, the pattern of device migration from one area to the other has to be modeled. This modeling method is known as mobile node mobility model. In mobile networks, like wireless sensor network, the nodes are commonly connected using mesh topologies. A mesh topology is important in modern wireless networks like wireless sensor network and MANET (the mobile adhoc network). This is because; the mesh topology improves availability in a network where each node in the network transmits data immediately after receiving a change in the physical environment.

A wireless mesh network has many important roles in modern communication to ensure availability of network service to the mobile nodes but, the performance of wireless mesh network is greatly affected by the mobility of the nodes [2]. In wireless local area network, the nodes are usually mobile nodes, like laptops, smart phones and tablets. These mobile nodes are connected to a stationery Access point. These Access Points connect thousands of mobile devices, like students' in a campus or passenger in airport and so on. The number of network users is increasing day by day, but the node mobility plays a major role in wireless local area network performance to provide ever resilient and highly available network.

Mobility in the perspective of wireless network is characterized by the route and the location of the mobile nodes and analysis of movement between Access Points in a network. When users migrate or move from one location to another location, then they de-associate and re- associate with Access Points in a network. The mobility model plays a vital role in characterizing the performance of wirless network [3, 4].

Another important characteristic of the wireless local area network is that nodes mobility is usually discontinuous in the time domain. In other words, a user can be associated with an Access Point let say X at 
time $\mathrm{t} 1$, then de-associates from this Access Point, and later on (at time $\mathrm{t} 2 \geq \mathrm{t} 1$ ) reappears in the network associated with another Access Point Y. This mobility pattern is very common [5, 6], for example, in a campus wireless network, where students' move from dormitory to classrooms, and turn off their laptops while moving from one location to the other.

The next generation wireless networks, such as the wireless sensor network and software defined wireless networks are required to be connected in mesh topology to ensure availability, unlike the traditional wireless network which is usually connected in the point-to-point or point-to-multi-point topology. The next generation wireless networks will greatly be affected by the node mobility because of the mobile equipment. Mobility has a great impact on the performance of the network. However, the node mobility models such as randomwaypoint and Gauss Markov have limitations [7]. The problem with randomwaypoint mobility model is that, it is non-smooth and Gauss Markovs model has non-stopping movement problems. The problem of non-stopping movement in Gauss Markov can be solved by dynamically controlling the motion from the controller in the software defined wireless networks.

\section{RELATED WORKS}

This section discusses, the research works, related to node mobility models in the software defined wireless mesh network. The next generation networks such as the wireless sensor network and software defined wireless mesh network [8-10] are required to be connected in mesh topology. The paper is focused on reviewing the literature.

In the traditional wireless network nodes are connected based on the point-to-point or point-tomulti-point topologies [11]. However, recently, the mesh topology is used in the wireless network. The mesh topology has become not as an option but, as a a requirement for the next generation wireless networks, such as the Wireless Sensor Networks, where nodes are required to transmit a data with peering devices. In such network the traditional topology the nodes require high availability ever resilient network to transmit the data or exchange the senor's data with the nearby nodes.

A wireless network mobility model describes, the mathematical model used to describe the motion of mobile nodes in a wireless network [12, 13]. Mobility model deals with two issues, the location management and handover management. Location management deals with monitoring the location of every node in the software defined wireless network and handover management deals with how nodes can be handed over from an access point to another access point. The handover decision can be made based on the signal strength or the load of access points. In signal strength based handover association method, nodes are associated to access points based on the strongest signal. This association method is referred as strongest signal first (ssf). In load based association method, the node is associated with access point with less overload compared to other access points in the range and is usually referred to as least load first(llf).

A study on wireless network mobility model shows randomwaypoint as a simplest mobility model and widely used in evaluation of MANET protocols [14-17]. This is due to its simplicity in implementation. This this model chooses a random destination and constant speed which the node uses to reach the destination. The parameters required for this model are, the maximum and minimum speed and the pause time.

Mobility models have a great influence on the performance of wireless mesh networks [18, 19]. This is because node movement may overload some Access points in the network while other access points are less overloaded or even no nodes are associated with them in the wireless network.

The node mobility models that are widely used in the software defined wireless network for real network node mobility emulation which are supported by the mininet-Wi-Fi, are the GaussMarkov [20-22], TruncatedLevy, RadomWayPoint, RefrencePoint, and TimeVariantCommunity model. And we will focus on the GaussMarkov node mobility model, which is discussed in detail in Section 3.

The Wi-Fi network, mobility modeling facilitates the handover management to enhance the network performance by de associating and re associating mobile nodes in the network from overloaded Access points to the less overloaded Access Points $[23,24]$ as they traverse through the access point coverage areas or regions.

\section{GAUSS MARKOV'S MOBILITY MODEL}

A Gauss Markov mobility model is used for simulating migrations of nodes in software defined wireless mesh network and this model used mainly in simulation. Mininet-WiFi supports the Gauss Markov's mobility model. The model commonly positions nodes in high mobility network. The three parameters used Gauss Markov's model are [4, 25-27], speed, direction and pause time. Each node in the network, updates its speed and direction during the pause time. And the model provides better authentic 
mobility of the nodes. Figure 1, shows the schematic diagram the node movement when the Gauss Markov's model is used in a network, with parameters $\max \_x=200, \max \_y=200$, speed $=20$ and time $=1$ ) and Figure 2 shows the node mobility using mininet-Wi-Fi graph. In the Gauss Markov model, the nodes are closely placed at random location in the virtual space and each node moves independently.
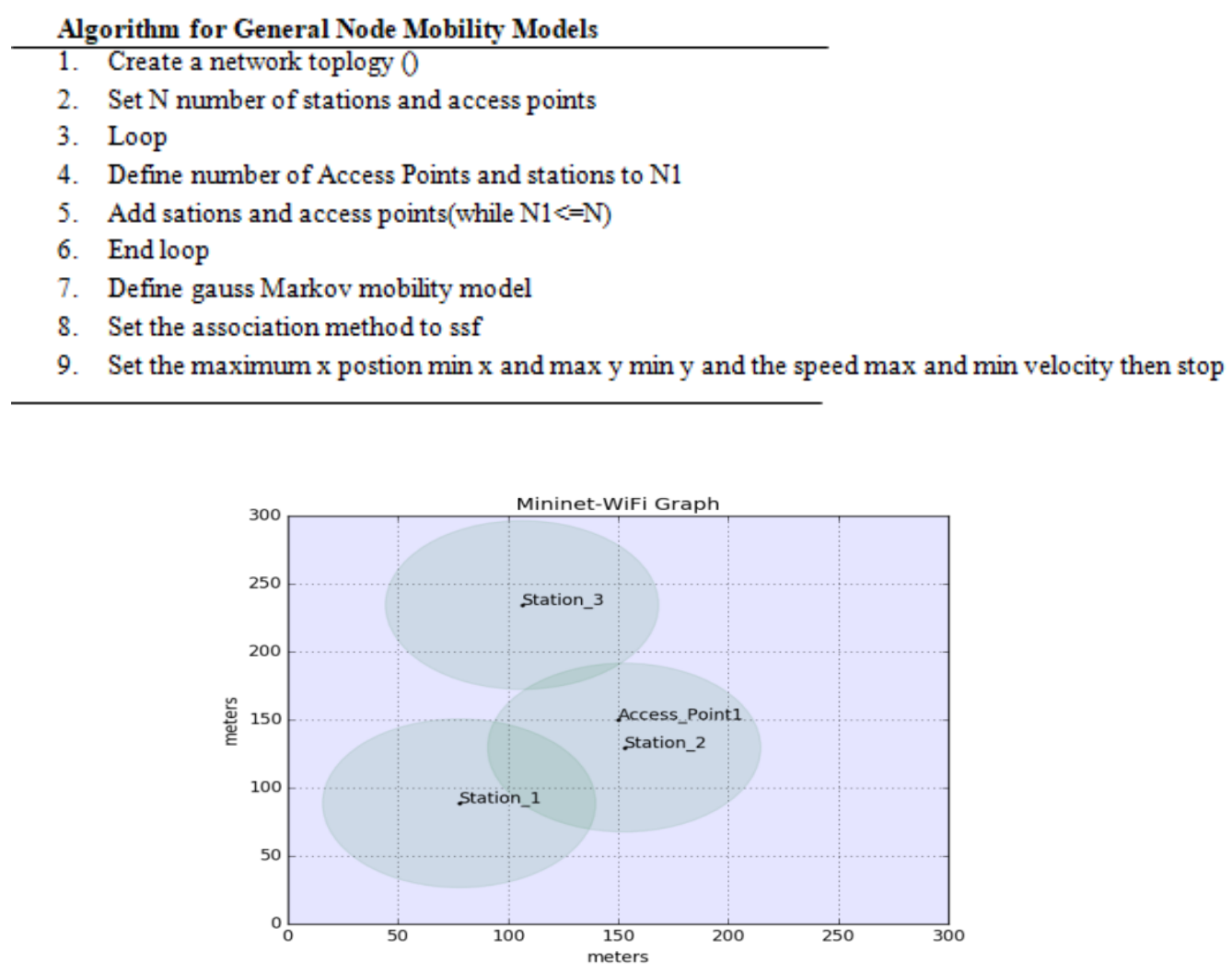

Figure 1. Gauss markov's node mobility model, mininet-wi-fi graph

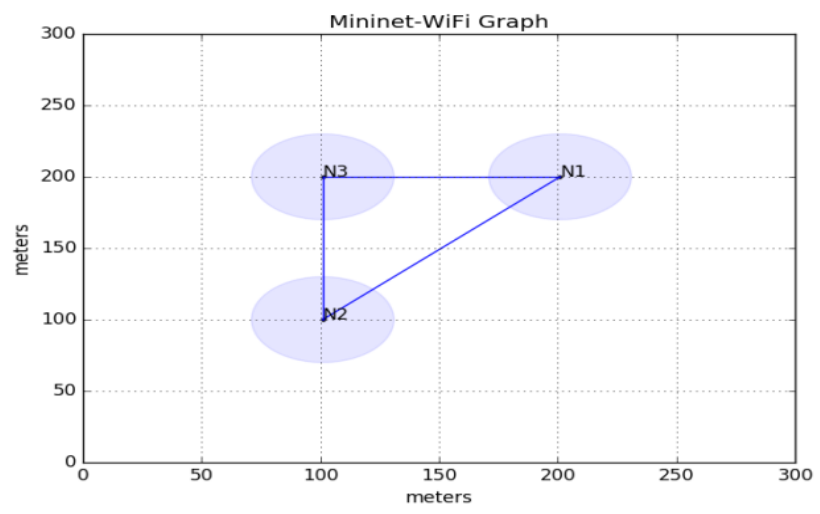

Figure 2. Software defined wirless mesh topology, with three nodes

\section{RESULTS AND DISCUSSIONS}

In this section, we have created a scenario where one station moves in a virtual space in the emulation enviroment, and where the node changes with access points, based on the strength of signal. The emulation is carried out using a mininet-wifi tool. For the emulation of Gauss Markov's mobility model we have created the mesh network topology consisting of three nodes, shown in Figure 3. 


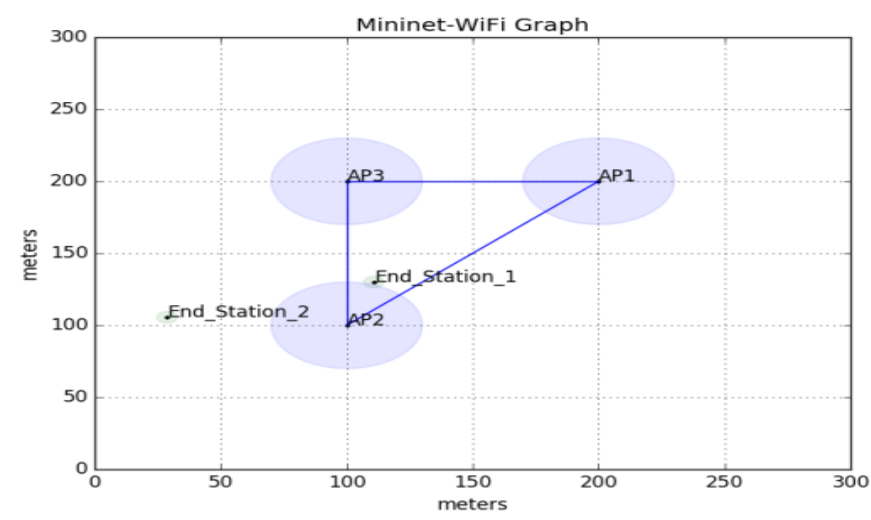

(a) Mobile nodes, Mesh Acess Points

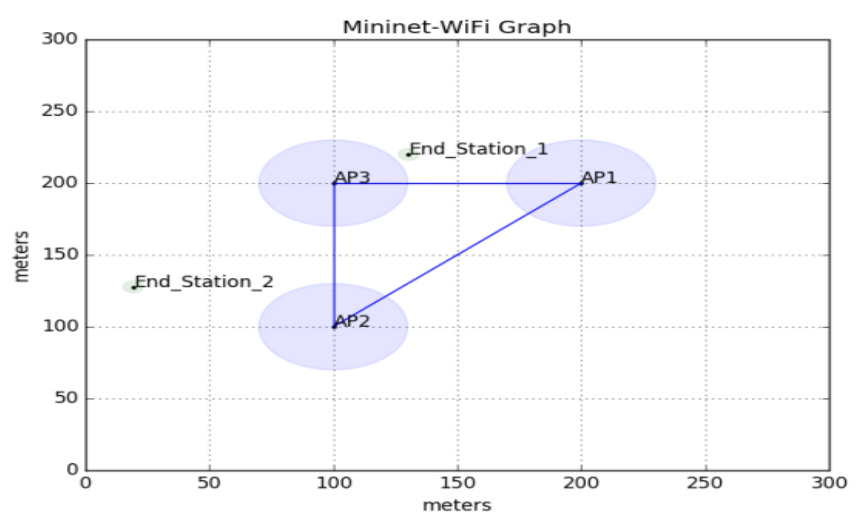

(b) signal strength based hand off

Figure 3. Gauss Markov's mobility model

\subsection{Mobility Based Handover}

To create a scenario where stations in the wireless network are mobile, we have set the range of the access points in mininet-Wi-Fi emulator. In mininet-Wi-Fi environment, we have used the strongest signal first (ssf) as the Association Control method. And the range of each access point was used to determine where handoffs occur between access points and which stattion may connect to which access point.

The mininet-WiFi enabled us to automatically move stations around in virtual space based on Gauss Markov mobility model. In the emulation, the GaussMarkov is used to move End_Station_1 and End_Station_2 around in an area 300 meters by 300 meters with a minimum velocity of 0.1 meters per second and a maximum velocity of 0.2 meters per second, using the python scrip shown in Figure 4(b).

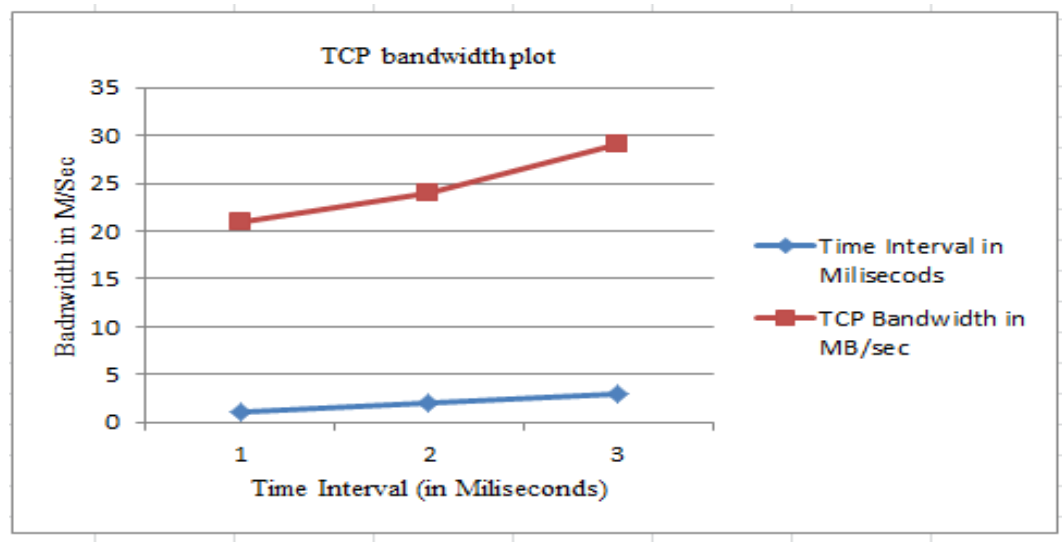

Figure 4. TCP traffic performance 
Figure 3 shows that the Mininet-WiFi implements scenarios with mobile stations that hand off between access points. Figure 3(a) shows mobile stations originally associated with access point, AP1 and Figure 3(b) shows the hand off End_Station_1 to access point, AP2. Mininet-WiFi automatically connects and disconnects End_Station_1 and End_Station_2 to and from access points, AP1 and AP2 based on either calculated signal strength. The hand off is determined based on strength of the signal, strongest signla first (ssf), association method is used in the above scenario. To verify that if the sation Sta1 is moved to access point AP1, we can use the following commands shown in (3) and (4).

Before handoff

mininet-wifi> sta1 iw dev sta1-wlan0 link

Connected to 02:00:00:00:03:00 (on sta1-wlan0)

After hand off SSID: ssid_ap2

mininet-wifi> sta1 iw dev sta1-wlan0 link

Connected to 02:00:00:00:04:00 (on sta1-wlan0)

SSID: ssid_ap1

\subsection{Software Defined Wireless Network Traffic Performance Evaluation}

To analyze the performance of Gauss Markov's mobility model in software defined wireless mesh network, we conducted detailed emulation using mininet-Wi-Fi free open source software defined wireless network emulator $[4,6,19,20,23]$. Then we have evaluated the performance of Gauss Markov's mobility model. The evaluation parameters used in the test bed environment are given in Table 1 and the performance result of TCP traffic between nodes measured using IPERF for the Gauss Markov's mobility model in Mininet-Wifi test bed environment is shown in Table 1.

Table 1. Parameters used in Performance Evaluation

\begin{tabular}{cc}
\hline Parameter & Value \\
\hline Communication range & $300 \mathrm{~m}$ \\
Speed & $1 \mathrm{~m} / \mathrm{s}$ \\
Number of Nodes & 2 \\
Packet Size & 114 bytes \\
\hline
\end{tabular}

\section{CONCLUSION}

In this work, we have emulated a software defined wireless mesh network using mininet-Wi-Fi and explored the Gaussian Mobility model which is widely used in software defined wireless network. The mininet-Wi-Fi tool is used to analyze and define the characteristics of user migration from access point to other access point. The experimental result showed that, the Gauss Makov's mobility model has a great influence on the network performance. The paper also explored how nodes migrate in a virtual emulation spaces using Gauss Makov's mobility model.

\section{REFERENCES}

[1] "Modeling and Simulation of CommunityMobility Model for Next Generation Wireless Networks using Coloured Petri Nets", 14th International Conference on Machine Learning and Applications, IEEE, 2015

[2] Abdul Hasib and Abraham O. Fapojuwo. "A Mobility Model for Heterogeneous Wireless Networks", IEEE 2008

[3] Nirmalkumar S Benni, Sunilkumar S Manvi. "Enhancement of data transmission using optimized multi cell approach in 5G backhaul wireless mesh network", Indonesian Journal of Electrical Engineering and Computer Science, Vol. 14, No. 1, April 2019

[4] Rupam Deb, Md. Morshedul Islam, Md Jashim Uddin, Jebunnahar, and Kazi Rafiqul Islam, "Performance Improvement of Seamless Vertical Handover in Heterogeneous Wireless Network", 2nd International Conference on Machine Learning and Computer Science(IMLCS'2013) August 25-26, 2013.

[5] Ming Zhao, Wenye Wang, "A Novel Semi-Markov Smooth Mobility Model for Mobile Ad Hoc Networks", Department of Electrical and Computer Engineering, North Carolina State University, 2015

[6] R.R. Roy, "Handbook of Mobile Ad Hoc Networks for Mobility Models", C Springer Science+ usiness Media, LLC 2011. 
[7] Meyanand.R.1, Ramya Dorai.D, "Analysis of Mobility Management in Wireless Mesh Networks using Random Walk Model", International Journal of P2P Network Trends and Technology (IJPTT) - Volume 1 Issue 2 September to October 2011

[8] Andrea Ribeiro, Rute C. Sofia, "A Survey on Mobility Models for Wireless Networks", SITI Technical Report SITI-TR-11-01 February 2011

[9] "Modeling Time-variant User Mobility in Wireless Mobile Networks", IEEE, 2007.

[10] Ramon R. Fontes, Samira Afzal, Samuel H. B. Brito, Mateus A. S. Santos, Christian Esteve Rothenberg, "MininetWiFi: Emulating Software-Defined Wireless Networks", IFIP, 2015.

[11] Elmano Ramalho Cavalcanti1, Marco Aurélio Spohn, "On the Interactions between Mobility Models and Metrics in Mobile ad hoc Networks", International Journal of Networks and Communications 2013

[12] DUAN Chenghao, “An Improved Routing Protocol Based on Gauss-Markov Model In Ad Hoc Networks Utilizing Prediction of Link Quality", Proceedings of the 34th Chinese Control Conference July 28-30, 2015

[13] Vinícius F.S. Mota, Felipe D. Cunha, Daniel F. Macedo, José M.S. Nogueira, Antonio A.F. Loureiro, "Protocols, mobility models and tools in opportunistic networks: A survey", Computer Communications, 2014.

[14] Zhaolong Ning, Qingyang Song, Yejun Liu, Fanzhao Wang, Xinyu Wu, "Markov-based vertical handoff decision algorithms in heterogeneous wireless networks", Computers and Electrical Engineering, Elsevier 2014

[15] Mallikarjuna Rao Yamarthy, M. V. Subramanyama and K. Satya Prasad, "A Multi-Layer Routing Protocol for Mobility Management in Wireless Mesh Networks", Twelfth International Multi-Conference on Information Processing-2016.

[16] Sabbir Ahmed, Gour C. Karmakar, Joarder Kamruzzaman, “An environment-aware mobility model for wireless ad hoc network", Computer Networks, 2010.

[17] Zeydin Pala, Kemal Bicakci, Mustafa Turk, "Effects of node mobility on energy balancing in wireless networks", Computers and Electrical Engineering, 2015.

[18] Tsehay Admassu Assegie, Pramod Sekharan Nair, "A review on software defined network security risks and challenges", TELKOMNIKA, Vol.17, No.6, December 2019.

[19] Tsehay Admassu Assegie, Pramod Sekharan Nair, "A review on software defined network security risks and challenges”, TELKOMNIKA, Vol.17, No.6, December 2019.

[20] Natarajan Meghanathan, "Impact of the Gauss-Markov Mobility Model on Network Connectivity, Lifetime and Hop Count of Routes for Mobile Ad hoc Networks" Journal Of Networks, Vol. 5, No. 5, May 2010 Yogita Shivaji Hande, M. Akkalakshmi, A Study on Software Defined Networking, International Journal of Innovative Research in Computer and Communication Engineering, Vol. 3, Issue 11, November, 2015.

[21] N. Padmavathya, Sanjay K. Chaturvedi, "Reliability Evaluation of Mobile Ad Hoc Network: With and Without Mobility Considerations", International Conference on Information and Communication Technologies (ICICT 2014).

[22] Sajal K. Das, "Mobility management A personal perspective”, Computer Communications, 2018.

[23] Tsehay Admassu Assegie, Pramod Sekharan Nair., "Performance analysis of emulated software defined wireless network", Indonesian Journal of Electrical Engineering and Computer Science, Vol. 16, No. 1, October 2019.

[24] Ricardo Silva, Jorge Sa Silva, Fernando Boavida, "Mobility in wireless sensor networks - Survey and proposal", Computer Communications, 2014.

[25] Song Xinchao, Zhao Yongsheng, Wang Lizhi, "Gauss-Markov-based mobile anchor localization (GM-MAL) algorithm based on local linear embedding optimization in internet of sensor networks", Cognitive Systems Research, 2018.

[26] Mincheol Kim, Seong-Lyun Kim, "Node Placement and Mobility Control in Mobile Wireless Sensor Networks", Proceedings of the 18th World Congress The International Federation of Automatic Control Milano (Italy) August 28 - September 2, 2011.

[27] Afonso Oliveira, Teresa Vazão, "Low-power and lossy networks under mobility: A survey", Computer Networks, 2016. 